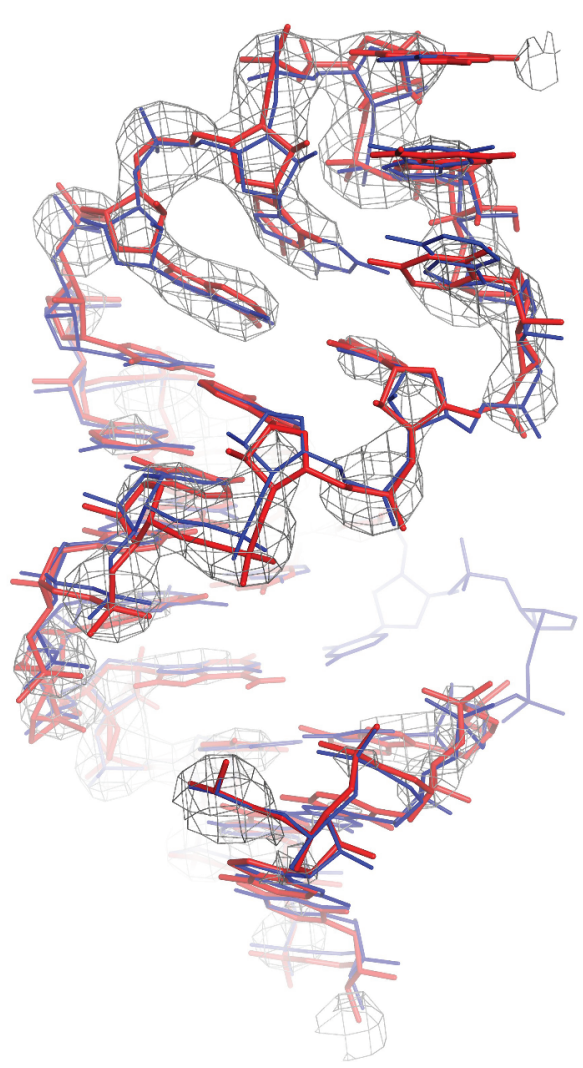

Figure 1. Comparison of published coordinates of the GCGA tetraloop from the group II intron IC subdomain (blue, PDB code 3bwp) and crystal structure model built using Brickworx (red). The model was fitted into the experimentally phased map $(3.1 \AA$ resolution) shown contoured at 3.0\%.

Keywords: model building, nucleic acids, macromolecular models

\section{MS4-P2 Advances in CCP4 software suite for macromolecular crystallography}

Andrey Lebedev ${ }^{1}$, Eugene Krissinel ${ }^{1}$, Charles Ballard ${ }^{1}$, Ronan Keegan $^{1}$, Ville Uski ${ }^{1}$, David Waterman ${ }^{1}$, Marcin Wojdyr ${ }^{2}$

1. CCP4, STFC, Research Complex at Harwell, Oxford, OX11 OFA, United Kingdom

2. Diamond Light Source Ltd, Harwell Campus, Oxford, OX11 ODE, United Kingdom

email: andrey.lebedev@stfc.ac.uk

The Collaborative Computational Project Number 4 (CCP4) was set up in the late 1970's in the UK to bring together developers of software for macromolecular crystallography. During the past years many leading software developers in the field of protein crystallography contributed to the CCP4, and the current CCP4 Software Suite provides tools and packages covering all aspects from data collection through to structure deposition [1]. Here we will present details of the latest release series of the Suite, version 6.5.

Release 6.5 brings a few new elements as well as updates and bug fixes to many of the components in the Suite. In particular, this release enforces ligand-related functionalities in CCP4 by introducing new structure and restraint generator Acedrg and carbohydrate validator Privateer-validate. In addition, the new tools enabled to curate and expand the monomer library. Advances in the processing part of the suite comprise processing multi-crystal datasets processing with Blend and multi-lattice datasets with Feckless software. Experimental phasing module has been significantly enforced with new automatic pipeline Crank-2.

In recent years, CCP4 Software Suite has undergone a considerable modernization, which made software building, testing, distribution and updating an automated routine. Yet new challenges arise owing to changing computing environments and concepts, OS updates, thirst for automation, increasing role of graphical front-ends and trends toward remote, web-based computations and hosting projects in the Cloud. We discuss these challenges and general directions for $\mathrm{CCP} 4$ development in middle-term perspective.

[1] M. D. Winn, C. C. Ballard, K. D. Cowtan, E. J. Dodson, P. Emsley, P. R. Evans, R. M. Keegan, E. B. Krissinel, A. G. W. Leslie, A. McCoy, S. J. McNicholas, G. N. Murshudov, N. S. Pannu, E. A. Potterton, H. R. Powell, R. J. Read, A. Vagin and K. S. Wilson (2011) Overview of the CCP4 suite and current developments, Acta Cryst. D67 235-242.

Keywords: Biological Macromolecular X-ray Crystallography, Software for Crystallography, Crystal Structure Determination 